\title{
Blast Overpressure Modeling Enhancements for Application to Risk- Informed Design of Human Space Flight Launch Abort Systems
}

\author{
Scott L. Lawrence, NASA Ames Research Center \\ Donovan L. Mathias, NASA Ames Research Center \\ Categories: Risk Assessment/Management, System Safety and Reliability
}

\section{SUMMARY}

This paper describes recent enhancements to the engineering-level analysis tools used by the Simulation Assisted Risk Assessment (SARA) project (Ref. 1) at NASA Ames Research Center in evaluating the blast overpressure risk to the crew. The primary enhancements to the model include incorporation of vapor cloud explosion (VCE) curve fits for propellant explosions, development of an improved model for the effects of vehicle velocity on blast propagation, improvement in the representation of blast/vehicle interaction effects, and incorporation of pressure vs. impulse (P-I) failure criteria to better represent structural failure modes. High-fidelity computational fluid dynamics (CFD) simulations, using the Overflow2 (Ref. 2) code, played a crucial role in the development of some of these enhancements. A subset of the high-fidelity results is presented.

\section{INTRODUCTION}

As part of an integrated launch vehicle ascent risk assessment program, data is required that characterizes the threat to the survival the crew associated with blast overpressure caused by catastrophic failure of the booster vehicle. The current work is intended to provide data toward understanding that threat. As shown in Fig. 1, blast overpressure is one of three main threats associated with catastrophic booster failure leading to explosion. The three threats are: 1) fireball radiant heating caused by relatively slow consumption of propellant, 2) blast overpressure associated with a shock wave generated by rapid burning (detonation or rapid deflagration) of propellant, and 3) relatively small, high-velocity fragments generated by confined-by-missile type explosions.

The bottom section illustrates, conceptually, the relative degree of the various threats and how they can vary with mission-elapsed-time (MET) due to changes in vehicle velocity and ambient pressure and density.

In order to apply the blast model pressure propagation information in a risk assessment, the blast overpressure and trajectory must be converted into failure probabilities. Simple engineering-level models were developed to model the blast overpressure environment and predict the risk to the crew. The models also provide additional data and insight into the design parameters that critically impact the ability to survive this failure mode. The components and inputs
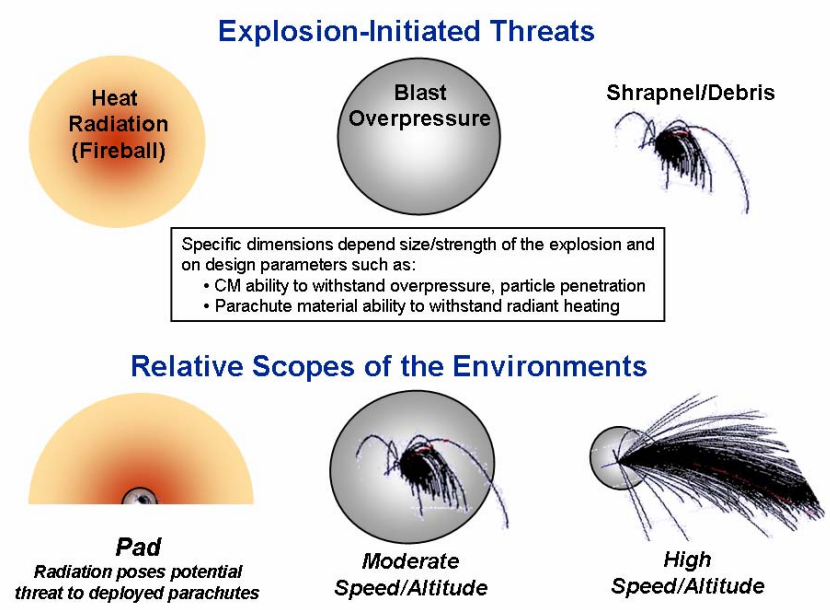

Figure 1. Types of explosion-related threats.

involved in the analysis of the blast overpressure failure mode are shown schematically in Fig. 2. The analysis couples models for explosive blast overpressure propagation with a simple LAV trajectory model to determine the overpressure experienced by the escaping crew module. This overpressure is then compared with the capsule's structural capacity to determine failure status. Both the blast and LEV propagation modules depend on multiple sub-components and inputs as shown in Fig. 2.

Most of the components and inputs are directly or indirectly dependent on the trajectory and the time of abort. The model assumes that the blast/crew capsule interaction can be represented by a relatively simple model, e.g., normal shock reflection. This assumption is understood to be of questionable validity and is the subject of substantial and ongoing computation fluid dynamics simulations, as will be subsequently discussed.

Currently, the ascent risk modeling is performed assuming two main classes of explosion environments: unconfined explosions following complete structural breakup and confined-by-missile (CBM) explosions. Data is generated for each of these explosion environments in the form of tables of failure probability as a function of mission-elapsed-time (MET) and warning time available. At present, unconfined explosions are assumed to lead to deflagration rather than detonation and are modeled as a Mach 0.75 vapor cloud 
explosion (VCE) using Baker-Strehlow-Tang families of curves (Ref. 3). Confined by missile explosions, on the other hand, are assumed to retain the potential for detonation and are represented using TNT blast characteristics.

\section{Explosion Modeling: Engineering-Level Model}

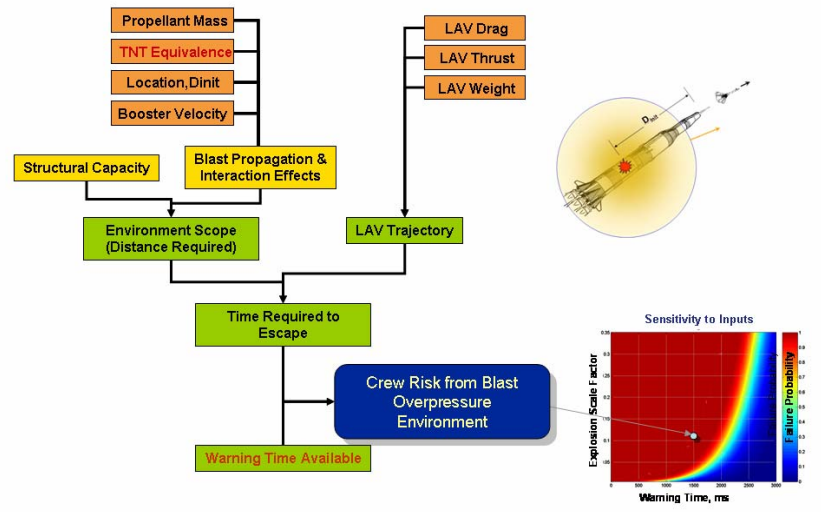

Figure 3. Schematic of blast model used for risk assessment.

The next sections describe the components in the engineering-level model, the high-fidelity analysis used to further understand the blast wave/vehicle interaction and a model for determining the survivability of the crew capsule to blast overpressure.

\section{ENGINEERING-LEVEL ANALYSIS}

\subsection{Blast Scaling and TNT Equivalence}

Distributions of blast parameters are typically expressed in terms of scaled coordinates and then "un-scaled" for specific situations. Two typical scaling methods are Hopkinson and Sachs scaling (see Ref. 4 for details on blast scaling). The most significant difference between the two methods is that the Hopkinson scaling assumes an ambient pressure, typically sea level, whereas the Sachs scaling enables scaled distributions to be applied to scenarios at various ambient pressures (or altitudes). For this reason, Sachs scaling is used in the engineering blast modeling tools developed within the SARA project. For a scenario-specific blast distribution, the altitude at which the blast occurs and the blast energy are required.

One of the most critical parameters that must be specified for a specific scenario is the blast energy, $E_{0}$. In the standard TNT equivalency approach, a yield factor is applied to the weight of available propellant representing the fraction of mass that is virtually converted to high-explosive (e.g., TNT). Mathematically,

$$
E_{0}=\eta_{\text {prop }} m_{\text {prop }} E_{T N T}
$$

Here, $\eta_{\text {prop }}$ is the yield factor, $m_{\text {prop }}$ is the mass of propellant available, and $E_{T N T}$ is the blast energy per unit of mass of TNT (here, $4.2 \mathrm{GJ} /$ ton is used).

The subscript on the factor $\eta$ is used to indicate that this factor has been considered a function of the propellant type. However, some research indicates this factor is mainly a function of the amount of surface area over which the fuel and oxidizer are in contact (degree of mixing). In any case, blast overpressure risk is extremely sensitive to the value of this parameter and it is well known to be difficult to specify because of its dependence on the specifics of the failure scenario.

Given a Sachs scaled distribution, scenario-specific blast distributions are developed by applying the blast energy and altitude to compute situation-specific dimensional distributions for peak overpressure and positive-phase impulse as functions of distance from the blast center. Shock relations are used to compute the blast arrival time from the peak overpressure distributions.

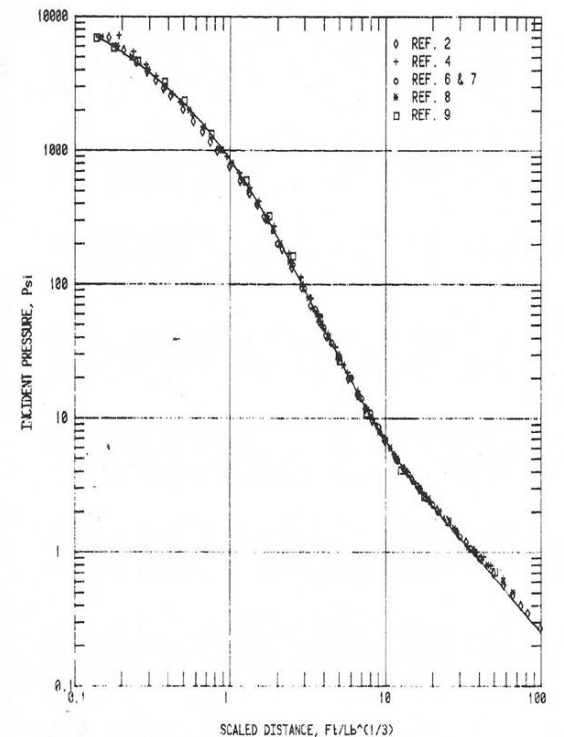

Figure 3. High-explosive overpressure distribution.

\section{2 "Standard" High-Explosive Distributions}

Engineering-level blast predictions are based on curve fits of high-explosive test data created by Kingery and Bulmash (Ref. 5). Curves for peak overpressure and positivephase impulse as functions of distances scaled using Hopkinson scaling are shown in Fig. 3. Also shown as symbols are the test data upon which the curves are based. The Kingery-Bulmash fits are considered a standard for highexplosive distributions, partly due to their incorporation into the Department of Defense models for blast overpressure and damage assessment (BlastX and CONWEP).

\subsection{Vapor Cloud Explosion (VCE) Distributions}

A recent enhancement to the SARA blast modeling methods is the introduction of the vapor cloud explosion families of curves due to Baker, Strehlow, and Tang (Ref. 3). Overpressure distributions for this family are plotted in Fig. 4. Use of these curves produces near-field blast behavior that better represents liquid propellant explosions than the TNT detonation model, which tends to over-predict the overpressure in the near-field. Various types of explosions can be modeled using the flame Mach number, $M_{f}$, to specify the member of the curve family on which the blast characteristic is based. The selected curve is then converted to a scenario-specific set of dimensional distributions using an energy equivalency and altitude in the same manner as the 
high explosives model.

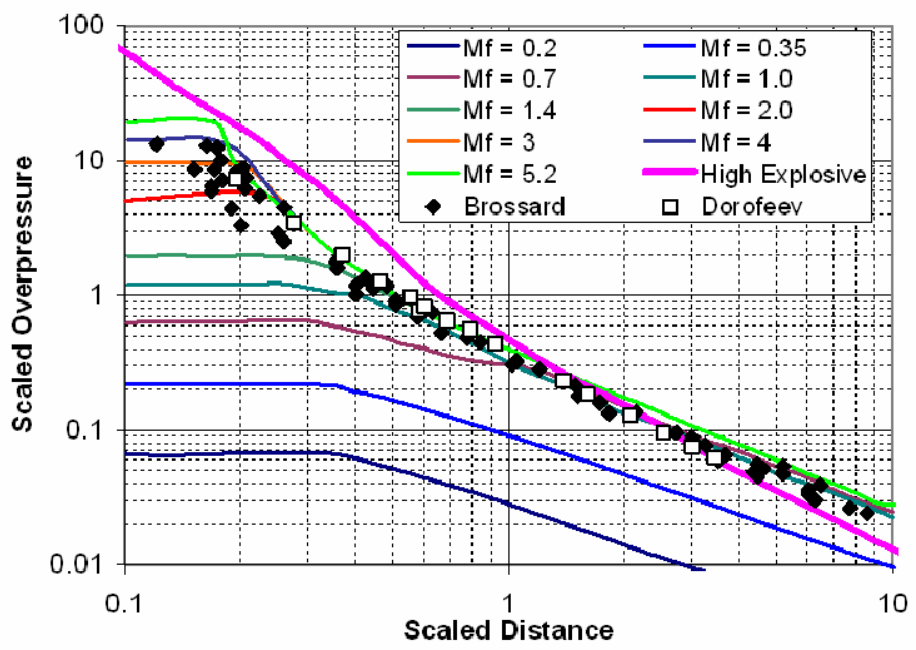

Figure 4. VCE overpressure distributions.

\section{CFD SIMULATIONS}

Computational fluid dynamic simulations have been performed in order to address a few of the deficiencies associated with the application of engineering-level blast propagation methods to launch vehicle explosion scenarios. In particular, the effect of vehicle velocity on the initial blast propagation process and the interaction between the blast wave and the escaping abort vehicle were investigated..

\subsection{Numerical solution process}

Blast propagation simulations were performed using the Overflow2 Navier-Stokes solver on an axisymmetric overset grid, shown in Fig. 5. (the subset of the grid shown contains every tenth radial point and every circumferential point). Overflow2 uses an overset structured gridding approach with information interpolated between overlapping subgrids at each time step. Time accuracy was ensured by using the dual-time-stepping option, in which several Newton iterations are performed at each time step.

Solutions were initialized by applying ambient flow everywhere except at points within an "energized sphere". The "energized sphere" is used to model the blast characteristics. For simulations that include the LAV and a freestream velocity, a steady-state solution for the flow over the LAV is computed prior to application of the energized sphere conditions.

\subsection{Modeling Effects of Vehicle Velocity}

One of the main objectives of the CFD simulations is to predict the effects of the oncoming freestream flow, produced by the vehicle velocity, on the blast propagation process. Initial simulations indicated that there are two primary effects: 1) an increase in the initial overpressure, and 2) an eventual stalling and blow-back of the blast wave as it

weakens. Results are shown in Fig. 6 for freestream Mach numbers of $0,0.5,1.0,1.5,2.0$, and 2.5.

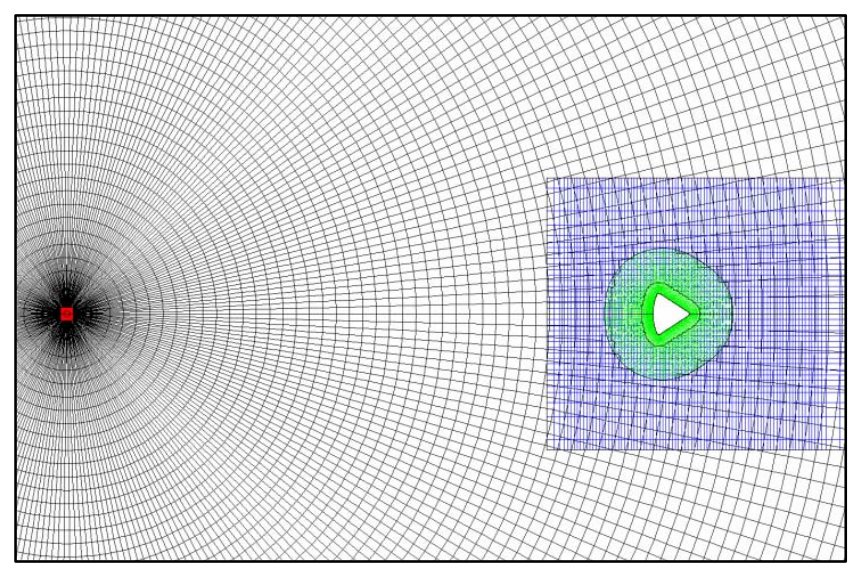

Figure 5. Computational grid for interaction simulations

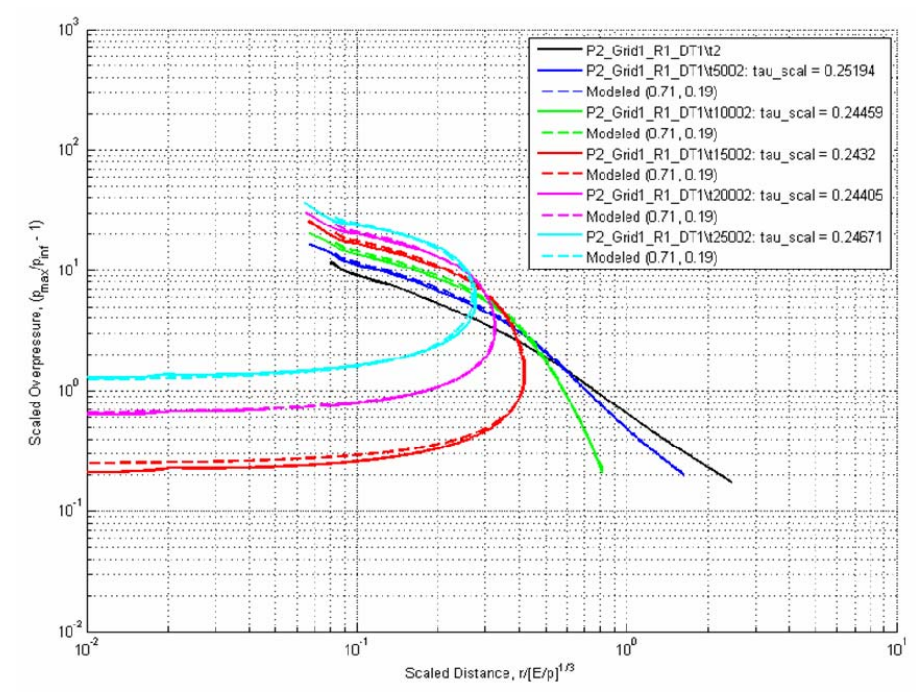

Figure 6. Crossflow effects on overpressure distributions

The initial attempt at including the vehicle velocity effects in the engineering-level model consisted of immediately freezing the center of detonation in inertial space at the time of detonation. While this modeled the blowback of the blast wave relative to the moving booster, it did not account for the initial elevated overpressure signatures and did not predict the total extent of penetration into the wind observed in the computations. The challenge was to represent the effects observed in the simulations in a simple enough method to include in the Excel/Matlab-based engineering model.

Such a model was developed using insight and data acquired from a number of CFD solutions. Simply stated, the model consists of a delayed blowback (relative to the booster) with an exponential growth in relative velocity. Results from this model are shown in Fig. 6 as the dashed lines. These curves were generated from the quiescent (black) curve using a simple formula based on vehicle Mach number. Agreement 
with the high-fidelity simulation data is considered to be very good.

\subsection{Simulation of Blast Interaction with Command Module}

A second objective of the CFD simulations was to compute the transient loads on the crew capsule that would be used for structural analysis of the capsule. The solutions were also used to assess the differences between the transient loading and the static overpressure loading. In order to illustrate the transient loading associated with the scenarios simulated, animations were generated for each case computed. This section presents key frames from one of the computed scenarios (5\%-yield blast wave at transonic propagation. As the blast encounters the reduced "headwind" in the vehicle wake, the portion of the shock in the wake is able to travel faster than the portion facing the full force of the freestream.

\subsection{Heat-shield reflection/influence factors}

As was illustrated in the previous subsection, interaction of the blast with the crew module creates pressure amplification effects associated with shock reflection from the heat-shield and refraction around the heat-shield shoulder. The interaction effects are quantified here in terms of "influence factors", or scale factors that can be applied to the undisturbed blast overpressure to obtain the overpressure felt

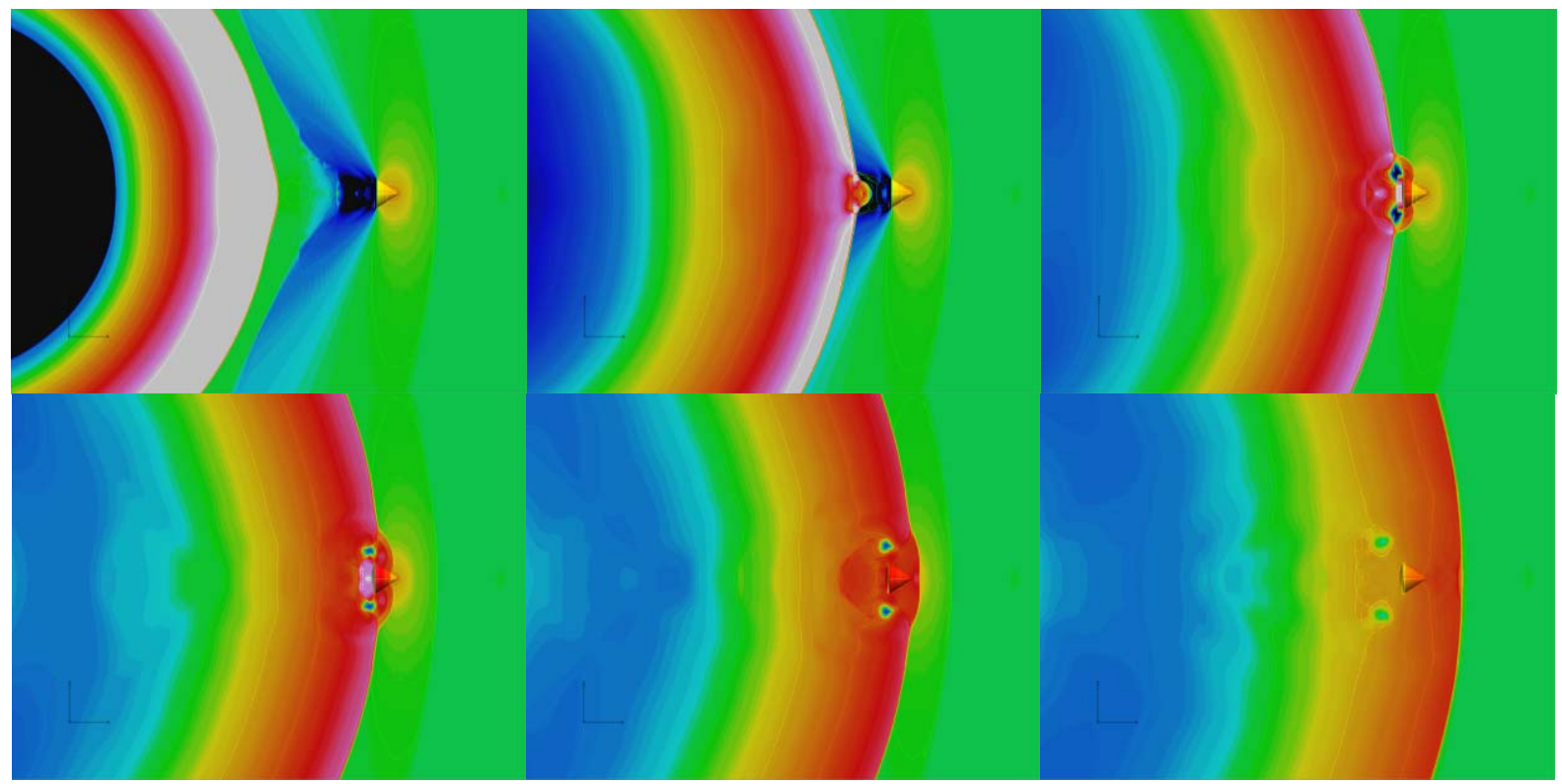

Figure 7. Snapshots of the interaction between a 5\%-yield blast wave and an LAV at transonic conditions.

conditions).

Six frames are presented in Fig. 7, representing snapshots of the blast at various phases of its interaction with the command module. Chronologically, the sequence goes from top left to top right and then from bottom left to bottom right. The parameter $\log \left(\mathrm{p} / \mathrm{p}_{\infty}\right)$ is plotted.

The simulation resolves the blast wave propagation toward the heat-shield of the crew capsule, reflection of the shock from the heat-shield surface, refraction of the blast wave around the shoulder and onto the conical aft surface of the command module, and redirection of the blast wave to the axial direction at the nose of the capsule. The pressure increased upon reflection from the heat-shield and decreased following refraction around the shoulder of the crew capsule. In this figure, white and red indicate high pressure and black and blue indicate low pressure.

The physics of the flight cases includes the effects of the momentum deficit in the wake of the capsule. The transonic condition provides a very long wake structure which results in some dramatic effects on the blast by the capsule. Of particular interest is the reflected overpressure at the heat-shield center. Figure 8 shows a plot of the overpressure ratio defined as

$$
\mathrm{IF}_{O P}=\mathrm{OP}_{\text {max }} / \mathrm{OP}_{\text {inc }}
$$

Here, $\mathrm{OP}_{\max }$ is the maximum overpressure felt at the specified location, i.e., the overpressure including the influence of the capsule, and $\mathrm{OP}_{i n c}$ is the overpressure taken from the unobstructed overpressure curve (e.g., Fig. 3) at the specified distance. In Fig. 8, influence factors for overpressure are shown for each of 23 simulations that were computed at various altitudes, Mach numbers, separation distances (between heat-shield and detonation center), and explosion strengths (yield factors).

For comparison, points are also plotted in Fig. 8 for the normal-shock reflection pressure for the given incident overpressure. In the initial engineering-level model, the influence factor was assumed to be equal to 2, i.e., a doubling of the incident blast overpressure. This is known to be accurate for weak (acoustic) waves; however, the highfidelity simulations made clear the need for a relation that 
would better represent reflection effects for stronger waves. The normal shock reflection relation, while somewhat inaccurate for stronger blasts at velocity and altitude, is generally slightly conservative and has been implemented in the current version of the engineering-level model.

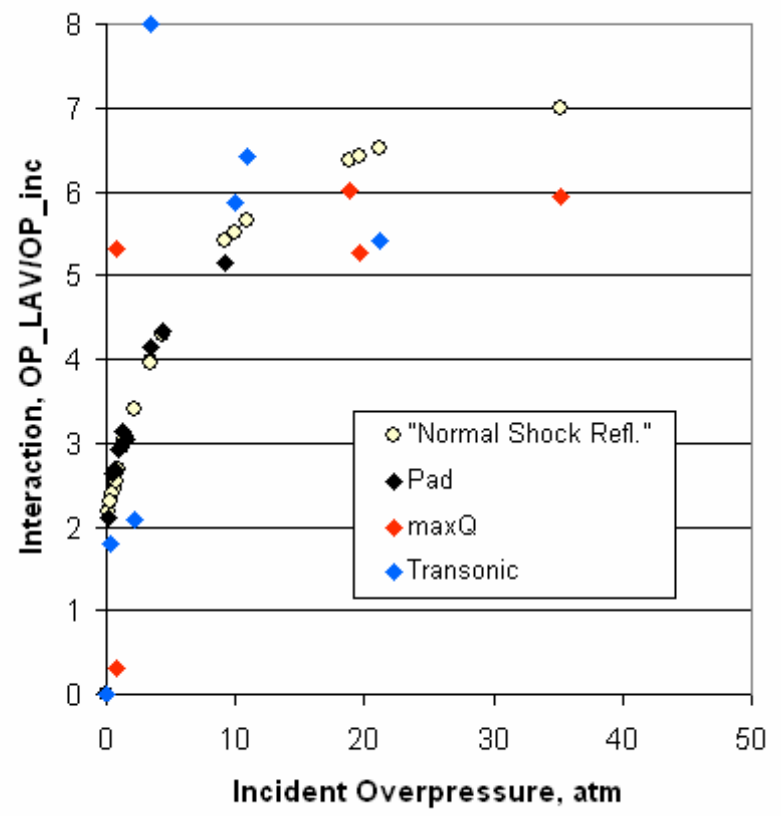

Figure 8. Interaction effects on heat-shield overpressure

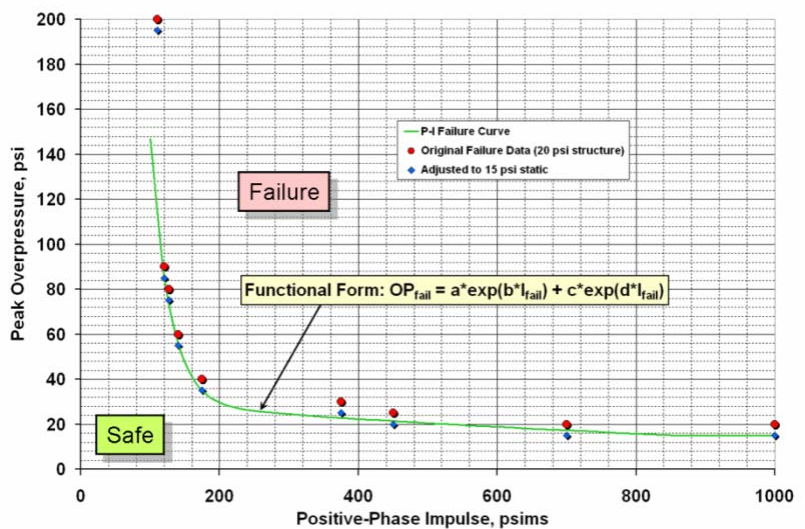

Figure 9. Overpressure vs. impulse structural failure diagram.

\section{STRUCTURAL RESPONSE}

Figure 9 shows results of some preliminary analysis intended to explore the potential value of a failure criterion that considers the manner in which structures respond to transient loading rather than using a static overpressure limit. The points on the curve labeled as "Original Failure Data" were determined based on structural analysis of a representation of the $\mathrm{CEV}$ command module structure. The curve is a two-term exponential function fitted to the data. While the analysis is very preliminary, the trends observed are consistent with those observed in the literature on structural response to transient loads (see Refs. 6 and 7).

What the data and literature indicates is that structures fail in different ways in response to transient loads, depending on the ability of the structure to absorb the pulse in the time that it is applied. Failure occurs for combinations of overpressure and impulse that fall above and to the right of the failure curve (green line). Large impulses applied with moderate levels of overpressure (far to the right) provide a "quasi-static" loading to the structure, whereas large overpressures at moderate impulse values (up and left), produce what are termed impulsive loadings. Note that the shape and location of the curve is dependent on the characteristics of the structure, specifically, its strength and natural frequencies.

\section{EFFECTS ON RISK INFORMATION}

Finally, the new model has been applied to explosion of the Saturn V $1^{\text {st }}$ and $2^{\text {nd }}$ stages, with Apollo launch escape system and two seconds of warning time. Results are shown for the new velocity effects model applied to high-explosive (KB-OP) and to a relative soft, flame Mach $=2$, VCE detonation (VCE_BST_OP). In addition, results are shown for the initial velocity effects model (Basic Blowback) with the high-explosive characteristic. Large counterbalancing effects are observed between the velocity effects model and the VCE model for this particular combination of inputs. Both the initial and current model indicate high risk at the pad $(\mathrm{MET}=0)$ that attenuates with time until the transonic condition near MET $=70$ seconds. The increased risk at this time is associated with the increased drag on the escape vehicle at this flight condition slowing the vehicle's separation.

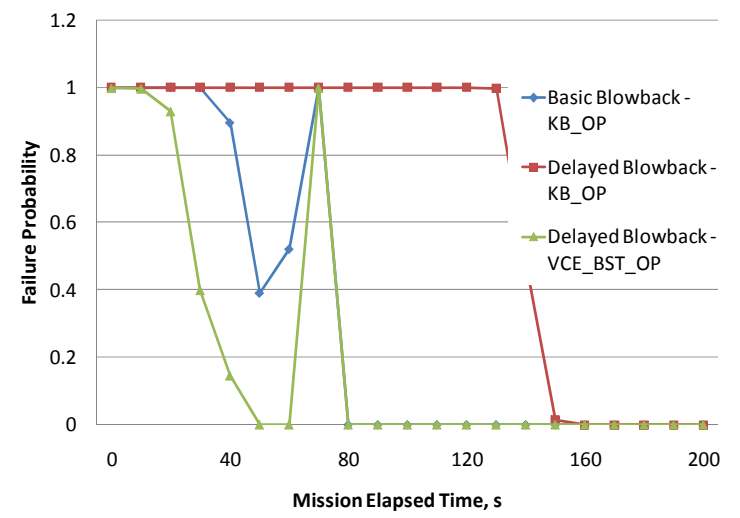

Figure 10. Saturn V/Apollo failure probabilities

\section{CONCLUSIONS}

An overview of the blast overpressure modeling approach used by the Simualtion Assisted Risk Assessment project has been described. Included in this description were a number of enhancements developed over the past year. These enhancements to a large degree were based on computational fluid dynamics simulations that provided qualitative insight into physical processes and quantitative calibration and validation of the new model components. Specific enhancements include a vapor cloud explosion model, an improved model for vehicle velocity effects, and an 
improved model for the effects of interaction of the blast and abort vehicle. These improvements have been observed to significantly alter the failure probabilities produced by the blast model and used by the risk assessment process, leading to subsequent improvements in the fidelity of loss-of-crew predictions. Work has begun on developing improved estimates of failure scenario-specific yield factors and development of CEV-specific P-I failure diagrams.

\section{REFERENCES}

1. Mathias, D., Go, S., Gee, K., and Lawrence, S., "Simulation Assisted Risk Assessment Applied to Launch Vehicle Conceptual Design," RAMS Paper 2007RM-300.

2. Buning, P. G., Gomez, R. J., and Scallion, W. I., "CFD Approaches for Simulation of Wing-Body Stage Separation," AIAA Paper 2004-4838, AIAA 22nd Applied Aerodynamics Conference, Providence, RI, Aug. 2004.

3. Tang, M. J., and, Baker, Q. A., "Comparison of Blast Curves from Vapor Cloud Explosions," J. of Loss Prevention in the Process Industries, 13, 2000, pp 433438.

4. Baker, W. E., "Explosions in Air," University of Texas Press, 1973.

5. Kingery, C.M., and Bulmash, C., "Airblast Parameters from TNT Spherical Air Burst and Hemispherical Surface Burst," Technical Report ARBRL-TR-02555, April 1984.

6. Baker, W.E., Kulesz, J.J., Ricker, R.E., Bessey, R.L., Westine, P.S., Parr, V.B., and Oldham, G.A., "Workbook for Predicting Pressure Wave and Fragment Effects of Exploding Propellant Tanks and Gas Storage Vessels," NASA Contractor Report 134906, September 1977.

7. Baker, W.E., Cox, P.A., Westine, P.S., Kulesz, J.J., and Strehlow, R.A., "Explosion Hazards and Evaluation,", Elsevier Scientific Publishing Co., 1983.
B IOGRAPHIES

Scott L. Lawrence

Mail Stop 258-1

NASA Ames Research Center

Moffett Field, CA 94035 USA

Scott.L.Lawrence@nasa.gov

Education:

PhD, Aerospace Engineering, Iowa State University, 1987.

Master of Science, Iowa State University, 1983.

Bachelor of Science, Iowa State University, 1981.

Professional Experience

1986-2002

Development and application of computational fluid dynamics codes for aerodynamic and aerothermodynamic analysis of supersonic and hypersonic flow.

2002-2004

Application of computational fluid dynamics to problems of crew safety, especially abort of winged reusable space launch vehicles.

2004-Present

Application of engineering level and high-fidelity physics modeling tools to problems relating to crew safety for capsule systems. Emphasis has been on determining failure environments created by catastrophic failure of launch vehicle leading to explosion.

Donovan L. Mathias

Mail Stop 258-1

NASA Ames Research Center

Moffett Field, CA 94035 USA

Donovan.L.Mathias@nasa.gov 